\title{
ECONOMÍA URBANA Y ECONOMÍA SOCIAL. UN RECONOCIMIENTO PENDIENTE ${ }^{1}$
}

\author{
Ruth Muñoz
}

\begin{abstract}
R E S U M E N La economía urbana (EU) surgió de la mano de propuestas espacialistas y economicismos y continúa siendo tallada por estas $A$ pesar de una evidente institucionalización de la economía social y solidaria (ESS) en América Latina, que da cuenta de un creciente pluralismo económico, en la EU perdura la hegemonía del enfoque del sector informal urbano y sus presiones por transformar en capitalistas cada práctica de economía popular. Buscando el reconocimiento de la economía social (ES) en este campo, el articulo ilustra brevemente la institucionalización de la ESS y plantea una provocativa exploración por poner en diálogo a la ES con la teoría de los dos circuitos. Un diálogo como este constituye una deuda pendiente que puede aportar a la disputa del campo así como a encontrar mejores comprensiones y, también, a transformar las economias de nuestros territorios en un sentido progresivo.
\end{abstract}

P A L A B R A S - C L A V E : economía urbana; dos circuitos de la economía urbana, economía social; economía social y solidaria; economía popular.

\section{INTRODUCCIÓN}

Este artículo busca el reconocimiento de la economía social (ES), inspirada en las prácticas de economía social y solidaria (ESS) por parte del campo de los estudios urbanos y, en particular, de la economía urbana (EU). A su vez, apunta a contribuir que se trascienda el recorte generalizado, fundamentalmente, a través de prácticas de la denominada ES tradicional o fundacional (cooperativas, mutuales y asociaciones) y sus aportes al desarrollo local.

Tanto las prácticas de la ESS como de la ES adquieren especial interés teórico y político en un contexto en el que la creciente insuficiencia dinámica del capital para absorber como mano de obra asalariada a la población económicamente activa y las estructuras económicas cada vez más marcadas por la financierización y la economía criminal agravan las condiciones de vida y de trabajo de las mayorías.

Sin embargo, tanto la EU como el desarrollo económico urbano y regional siguen tallados por propuestas espacialistas, fundamentalmente, desarrolladas en EEUU o Europa, que poco se preocupan o entienden ${ }^{2}$ nuestras economías latinoamericanas, que son adoptadas de forma bastante acrítica en América Latina y que, como sostiene Cuervo (2005), toman como referencia ciudades y territorios de los países desarrollados que constituyen falsos espejos para la ciudad latinoamericana. ${ }^{3}$

A su vez, observamos como destacable la falta de evidencia empírica sobre los logros que tienen estas teorías al implementarse en políticas públicas en
1 Este trabajo toma como base la ponencia "Heterogeneidad estructural de las metrópolis latinoamericanas. Una revisión que busca el dialogo entre los principales abordajes", presentada en el 1er. Congreso de estudios urbanos: "Pensar la ciudad, cambiar la ciudad Los retos de la investigación en América Latina a comienzos de siglo XXI". Organizado por el Programa Universitario de Estudios sobre la ciudad (Instituto de Investigaciones Sociales-UNAM); el Instituto del Conurbano (UNGS) y la Revista Mundo Urbano. Los Polvorines, agosto de 2011.

2 Véase, por ejemplo, Cimoli, M.; Primi; A.; Pugno, M. (2006). "Un modelo de bajo crecimiento: la informalidad como restricción estructural". Revista de la CEPAL n. 88. Donde rápidamente, antes que buscar entender los procesos que denominan "informalidad", le adjudican el papel de ser una de las principales causantes del bajo crecimiento de nuestras economías!

3 Sin embargo, vale la pena señalar que esto no es propio sólo de las teorías urbanas y regionales sino del pensamiento económico de la periferia en general, como da cuenta Sztulwark (2003) al señalar que el nuevo estructuralismo latinoamericano, a diferencia del de posguerra (que tiene a Prebisch y la CEPAL de los inicios como sus principales exponentes), denota una mayor preocupación por los aspectos profesionales (instrumentales y metodológicos) que brindan "legitimidad académica" al economista con fuertes presiones por el formalismo; una pérdida de radicalidad $y$, también, de originalidad al debilitarse el rasgo propiamente latinoamericano de los aportes teóricos. Para más detalles, véase: Sztulwark, S. (2003). El estructuralismo latinoamericano. Fundamentos y transformaciones del pensamiento económico de la periferia. Serie Informes de Investigación n. 16. Los Polvorines, Instituto de Industria, UNGS. 
territorios concretos o los resultados que efectivamente alcanzan. Al respecto, desde la perspectiva de la ES es preciso recordar los hallazgos de Fernández y Vigil (2009) que, al evaluar políticas de clusters en Argentina, expresaron que era posible alcanzar una competitividad regional sin inclusión social ni mejora de las condiciones de vida.

En lo que sigue, se presenta brevemente a la ES como una subdisciplina y, luego, se ilustra la institucionalización de la ESS en América Latina, poniendo atención sobre algunos procesos organizativos urbanos. A continuación, se problematiza la vigencia del enfoque de informalidad y, posteriormente, se plantea un necesario diálogo entre la teoría de los dos circuitos de la economía urbana en los países subdesarrollados y la ES. Finalmente, se esbozan algunas reflexiones.

\section{LA ECONOMÍA SOCIAL EN SU CARÁCTER DE SUBDISCIPLINA}

La ES como subdisciplina, en construcción, se nutre fundamentalmente de la antropología económica, la sociología económica, la historia económica y la economía política, para abordar "lo económico" como un objeto pluridisciplinario en el que los principios de institucionalización en la sociedad (categoría de K. Polanyi) se vuelven centrales para comprender la realidad abordada y lograr salirse de los economicismos hegemónicos de la literatura económica actual.

De todas formas, excede el alcance del artículo presentar la ES. Apenas expondremos algunas categorías centrales de Coraggio e iremos aplicándolas en los puntos subsiguientes. Sin embargo, vale la pena remarcar que varios autores han realizado un camino dialéctico desde las problemáticas urbanas latinoamericanas a la ES, entre los que se destacan además de Coraggio, Singer, Quijano y Federico Sabaté. ${ }^{4}$

En este marco, se define la economía de las metrópolis latinoamericanas como una economía regional mixta, abierta y en posición dominante con el resto del país y de dependencia con el exterior, siendo una característica sustantiva la hegemonía capitalista y la incompletitud del desarrollo del capital, en tanto subsiste un gran sector de economía popular con trabajo organizado de forma autónoma. Por tratarse de una región central, es preciso complejizar el sector externo de la economía mixta, teniendo en cuenta tanto las relaciones con el resto del mundo como las que establece con las provincias. ${ }^{5}$

Al esquema tradicional de dos subsistemas (Mercado-Estado; privadopúblico), Coraggio (2011a) contrapone un abordaje de la economía mixta con tres subsistemas o sectores, jerarquizando por sobre otros (como el tamańo o la propiedad de los medios de producción) el criterio de la racionalidad que sirve para reagrupar los subsistemas y dar cuenta de las diversas unidades económicas, recursos y relaciones intra e intersectoriales a través de las que cada sociedad resuelve lo económico. Encontramos así, por un lado, la economía empresarial capitalista, cuya principal unidad organizativa es la empresa privada, orientada por la acumulación privada de capital; por otro, la economía pública, asentada en la práctica económica de los distintos niveles estatales y las empresas públicas, 
orientados por una combinación de necesidades sistémicas, muchas veces contradictorias, como el bien común (cuya definición es materia de lucha social) y la gobernabilidad del capital; y, finalmente, la economía popular, orientada por la reproducción de las vidas de los miembros de las unidades domésticas, grupos y comunidades. Aquí, se entiende por economía popular la economía de los trabajadores, es decir, de aquellos miembros de la sociedad que dependen de la realización de sus capacidades de trabajo para obtener su sustento. Este se da a través de la combinación del trabajo para la producción de satisfactores de consumo doméstico, el trabajo para la producción de bienes o servicios para la venta en el mercado y el trabajo organizado por patrones que contratan a los trabajadores como fuerza de trabajo por un salario ya sea estatal o privado. En este esquema, la ESS se encuentra en la intersección de los tres sectores, con un fuerte componente en la economía popular organizada y la racionalidad de la reproducción ampliada de la vida de todos (y no solo de sus miembros).

Hasta aquí la presentación básica, destacando que el campo de prácticas de la ESS que trataremos a continuación cuenta con una diversidad de interpretaciones, siendo los desarrollos de Coraggio distintivos debido a que, entre otras cuestiones, analizan profundamente las prácticas de economía popular y la valoran como un punto de partida, aunque no el único, para una estrategia política en territorios concretos.

\section{SOBRE LAS PRÁCTICAS DE ESS: OTRA ECONOMÍA Y OTRA CIUDAD SON POSIBLES}

La institucionalización de la ESS en América Latina es evidente. Nos concentraremos en este artículo en dicho proceso desde el punto de vista del Estado, dejando para otros trabajos la institucionalización a raíz de las prácticas alternativas de la sociedad civil. Aunque, en gran parte, estaremos dando cuenta de ellas en virtud de que (salvo claramente en el caso de Venezuela) el accionar del Estado apunta a dar reconocimiento y respuestas a prácticas existentes en la sociedad civil en relación con la ESS y, por tanto, hablar del Estado es hablar de ellas mimas. Incluso, más allá de que se identifiquen y/o se autodenominen como tales o que no lo hagan y que, desde nuestro punto de vista teórico, pueden ser categorizadas de esta forma (en virtud de su probada racionalidad por la reproducción ampliada de la vida).

Aproximadamente, en los últimos diez años, varios países han implementado políticas y/o programas, creado instituciones específicas y hasta establecido nuevas constituciones que dan reconocimiento a las prácticas de la ESS y las conciben como sujetos de derecho.

Al respecto, en los casos de Argentina, Brasil, Venezuela, Bolivia y Ecuador, Coraggio (2011b) señala que, en términos generales, se encuentran diferentes niveles de problematización sobre qué es lo económico y cuál es el rol del Estado y los mercados; con Estados que están recuperando su papel interventor a través de orientaciones "nacionales y populares" y que avanzaron en cierto distanciamiento (en distinta medida según los casos) de las políticas más tradicionales de promoción del autoempleo y la microempresa informal propias de la PREALC-OIT.
6 Muñoz, R. (2013). "Políticas económicas poscrisis. Aprendizajes y desafíos a partir de la institucionalización de la economía social y solidaria en América Latina". Capítulo elaborado en base a la presentación realizada en la Table ronde "Créer de nouvelles politiques", en el marco del Colloque international "Penser et construire de nouveaux référentiels écononomiques", organizado por el Ministerio Delegado de la economía social y Solidaria. Bercy, París, 5 de febrero de 2013 y sobre el cual actualmente se está editando un libro. Dado que circulará en francés, se retoman aquí algunas cuestiones allí desarrolladas. 
714 de las 24 provincias argentinas crearon en los últimos años unidades organizativas vinculadas a la ESS y lo mismo hicieron las ciudades de Buenos Aires, Córdoba y Rosario; la mayoría de estas instancias tiene el rango de Direcciones y están ubicadas bajo las órbitas del "Desarrollo Social". Para más detalles, se sugiere la lectura de: Hintze, S., Deux Marzi, M. V., Costa, M. I. (2011). "Los organismos públicos de promoción del trabajo asociativo autogestionado en la Argentina" en Hintze, S.; Danani, C.; (coords.). Protecciones y desprotecciones: la seguridad social en Argentina 1990-2010. UNGS, Los Polvorines; disponible en: < $\underline{w} \mathbf{w}$. ungs.edu.ar>

8 En Muñoz (2013) calculamos en base información del Instituto Nacional de Asociativismo y Economía Social (INAES), en Argentina hay 21.168 cooperativas, de las cuales el $71,4 \%$ son de trabajo, las cuales crecieron más de 13 veces desde el 2003 a la actualidad, resultando de gran impacto el Programa Ingreso Social con Trabajo (PRIST), creado en agosto de 2009 que propone la creación de cooperativas de trabajo de alrededor de 60 trabajadores cada una para la ejecución de pequeñas obras de infraestructura (http:// www.desarrollosocial.gov. ar/ingresosocialcontrabajo/114).

9 Que como explica Coraggio en el trabajo en el que nos estamos basando (2011), el buen vivir es la forma que asume la reproducción ampliada de la vida en la constitución ecuatoriana, lo que implica orientar el sistema económico de manera que permita lograr cuatro equilibrios: el equilibrio de los seres humanos consigo mismos; el equilibrio entre los seres humanos; el equilibrio de los seres humanos con la naturaleza; y el equilibrio entre las comunidades de seres humanos. Una expresión de esto en términos de política pública, se puede encontrar en el Plan Nacional para el Buen Vivir (http://plan. senplades.gob.ec).
Resumimos muy sucintamente los casos de Argentina, Brasil y Ecuador, seleccionados por la mayor cercanía a nuestra investigación y para dar cuenta de la diversidad de contenidos que hacen a dicha institucionalización. Para hacerlo, nos basaremos en el trabajo de Coraggio (2011b) y en el de Muñoz (2013). ${ }^{6}$

A la institucionalización de la ESS en Argentina el autor la denomina como una "política social de economía social" que tomó fuerza luego de la crisis 2001-2002, con una orientación asistencialista aunque con cambios simbólicos que plantean un pasaje de "beneficiarios" a "sujetos de derecho"; muchas veces vinculada a la categoría de "desarrollo local" en programas, planes y nuevas instituciones burocráticas; ${ }^{7}$ siendo el Ministerio de Desarrollo Social de la Nación el principal actor que promueve esta institucionalización con movimientos sociales y sindicales crecientemente fragmentados.

Los sujetos jerarquizados en tanto destinatarios de estas políticas son emprendimientos mercantiles populares o asociativos de muy baja escala, con una creciente focalización en los sectores empobrecidos y/o con problemas de empleo y, en los últimos años, con el "nuevo" cooperativismo de trabajo que reconfigura el mundo cooperativo del país, ${ }^{8}$ a partir de lo que Vuotto (2011) denomina cooperativismo inducido por el Estado.

A nivel del diseño de las políticas, son comunes las consultas a los destinatarios, aunque no siempre son vinculantes. A su vez, a nivel de implementación, hay una gran participación de diversos movimientos sociales, especialmente los más afines políticamente al gobierno. Son destacables las políticas de gestión asociada que promueven la ley de microcrédito.

Por su parte, al caso de Brasil Coraggio lo categoriza como "co-construcción de políticas públicas para la economía solidaria”, que promueven el apoyo, la ampliación y los encadenamientos de los emprendimientos del sector de economía solidaria, con énfasis en sus componentes mercantiles, siendo dichas políticas, en su mayoría, construidas con la participación de los movimientos de economía solidaria.

Se observa una institucionalidad muy interesante, entre la que se destaca la creación de la Secretaría Nacional de Economía Solidaria (SENAES) en el marco del Ministerio de Trabajo y Empleo (aunque la economía solidaria aparece en el discurso de varios ministerios y políticas), el Foro Brasileño de Economía Solidaria (FBES), la creación del Consejo Nacional de Economía Solidaria (CNES) como órgano consultivo y propositivo, el Sistema Nacional de Comercio Justo y Solidario siguiendo la demanda de la II Conferencia de Economía Solidaria (CONAES) y los mapeamentos realizados fundamentalmente por la red UNITRABALHO.

En general, se trata de políticas de mayor alcance y más estructurales que en el caso argentino aunque, desafortunadamente, investigaciones recientes arrojan que (tanto en el caso de Brasil como en el de Venezuela no abordado en este artículo), las prácticas no son tan intersectoriales ni transversales como sostienen los discursos (HINTZE, 2010).

Por último, la institucionalización de lo que denominan como "economía popular solidaria”, en Ecuador, es presentada por Coraggio (2011b) como una propuesta "hacia un sistema económico social y solidario", que se ve plasmado en la Constitución de Montecristi (2008) que tiene el horizonte puesto en el buen vivir (traducción del Sumaq Kawsay en quechua). ${ }^{9}$ Esta concepción plantea una nueva relación entre economía, sociedad y naturaleza, la cual establece un uso racional de los 
recursos naturales, cambia las reglas de su apropiación (por ejemplo, el agua no puede ser privatizada) y establece a la naturaleza como sujeto de derechos. ${ }^{10}$

Es en este marco que se da un reconocimiento al pluralismo económico, a través de diversas formas de organización económica: pública, privada, mixta, popular y solidaria. Por su parte, el sector económico popular y solidario está conformado por las organizaciones de los sectores cooperativo, asociativo y comunitario, y las unidades económicas populares (abarcando en ellas a las que se dedican a la economía del cuidado, los emprendimientos unipersonales, familiares, domésticos, comerciantes minoristas y talleres artesanales).

A su vez, en Ecuador encontramos una aplicación directa de estos contenidos de la constitución en marcos normativos más específicos, como son la Ley Orgánica de la Economía Popular y Solidaria y del Sector Financiero Popular y Solidario (2011) y su reglamento (2012). Esta normativa fortalece instituciones estatales preexistentes como el Instituto de la Economía Popular Solidaria (IEPS) en el marco del Ministerio de Inclusión Económica y Social (MIES) y da lugar a la creación de nuevas instituciones como la Superintendencia de Economía Popular Solidaria (SEPS).

Teniendo en cuenta estas institucionalizaciones, con las diversas denominaciones, alcances y orientaciones, pondremos a continuación más atención a procesos más específicamente urbanos. Si bien en la literatura urbana se suelen valorar las experiencias de economía popular, fundamentalmente, prácticas de autoconstrucción de la vivienda y mejoras de asentamientos, villas y barrios populares, es llamativamente dispar el reconocimiento a iniciativas que han superado el horizonte de la supervivencia y que dan muestras del potencial de la ESS a nivel socioeconómico.

Pese a las construcciones de barrios enteros por parte de movimientos sociales (organizados, en general, bajo diversas formas de cooperativas) que exceden ampliamente la satisfacción individual de las necesidades; y pese a la recuperación de empresas por parte de sus trabajadores y la densificación de las cadenas en las que participan, el campo de la EU sigue negándolos, dándoles un tratamiento marginal o abordándolos con marcos teóricos inadecuados.

¿Con qué conceptos teóricos legitimados en el ámbito de la EU se pueden explicar casos como la cooperativa de servicios públicos integrales "Comunidad Organizada. Unidos para Crecer" Ltda. ?' ${ }^{11}$ Se trata de un caso en cuya génesis estuvo la organización de los vecinos de Cuartel V de Moreno (oeste del Conurbano Bonaerense - Argentina) para el armado de un fideicomiso a través del cual financiaron la instalación del gas natural en toda la localidad. Luego, llevaron adelante el armado de cooperativas de trabajo en el marco del PRIST (ver nota al pie 9), para el mejoramiento barrial y la promoción del trabajo. Actualmente, están enfrentando una seria problemática en torno a la gestión de la basura. Estas acciones, entre otras, dan cuenta de sus principios, motivaciones y racionalidad en pos de la reproducción ampliada de la vida de todos.

Apelar a categorías como el capital social de los pobres o seguir poniendo la lupa en la autoconstrucción doméstica, el autoempleo o el acceso a la ciudad a través de los llamados mercados inmobiliarios informales, sin dar cuenta al mismo tiempo de iniciativas colectivas como esta, obstaculiza el enriquecimiento de abordajes a procesos urbanos que tienen vocación de cambio y el reconocimiento de experiencias que ameritan ser jerarquizadas como sujetos y objetos de estudio y acción política en el campo de los estudios urbanos, en general, y de la EU, en particular.
10 En relación con este tema, se sugiere profundizar con: Acosta, A. (2010). El buen vivir en el camino del postdesarrollo. Una lectura desde la Constitución de Montecristi. Policy Paper 9, Friedrich Ebert Stifgtung, Quito. Disponible en: <http://library. fes.de/pdf-files/bueros/ quito/07671.pdf>.

11 Se sugiere la siguiente tesis de la Maestría en economía social (ICO-UNGS) que analiza, entre otros casos, el de esta experiencia: Mutuberría Lazarini, V. (2007). "Los servicios públicos urbanos como medios colectivos para la producción y reproducción de la vida de los sujetos en sociedad desde la perspectiva de la economía social. Análisis de experiencias de gestión colectiva en el Gran Buenos Aires". Disponible en: <http://www.ungs. edu.ar/cm/uploaded files/file/posgrados/tesis/ Trabajo final tesis Maestria.pdf>. 
12 Coincidíamos entonces con Lopes de Souza (2004) cuando señalaba que los analistas de la reforma urbana examinan la reproducción de la fuerza de trabajo en términos estrechos y que no tratan, o lo hacen de forma muy marginal, la esfera de la producción. También estábamos de acuerdo con su planteo que apuntaba a "la división del trabajo académico existente en las ciencias sociales entre geógrafos, arquitectos, sociólogos y economistas que superenfatizan aspectos parciales de un mismo problema general" (Lopes de Souza, 2004: 38) como una de las causas de la ignorancia recíproca que observó entre los promotores de los presupuestos participativos, la reforma urbana y la economía popular, tan evidentemente imbricados entre sí. Se sugiere la lectura de este interesante artículo: Lopes de Souza, M. (2004). "Reforma urbana, orçamentos participativos e economia popular: relaciones de complementaridade" em Revista Ciência e Cultura. v. 56, n. 2. Disponible en: <http:// cienciaecultura.bvs.br/ scielo.php?pid=S00096725 2004000200019\&script $=$ s ci_arttext>.

13 Disponible, por ejemplo, en: www.hic-net.org/news. php?pid=4329

14 Por ejemplo, véase el "Encuentro Intercultural por el Buen Vivir del Campo y la Ciudad", disponible en: <http:// pichinchaporelsi.blogspot. com.ar/2011/04/encuentro-intercultural-por-el -buen.html>.

15 Se recomienda la lectura de Hart, K. (2012). "How the informal economy took over the world", disponible en la página que tiene al autor como uno de los dos administradores: <http://thememorybank. co.uk/2012/10/17/the-informalization-of-the-world-economy/>. En la actualidad, Hart está acercándose a la ES, siendo muestra de ello el libro co-editado con reconocidos autores del campo: Hart, K.; J. L. Laville; Cattani, A. D. (2010). The Human Economy. A Citizen's Guide. London, Polity Press.
Por otro lado, en línea con la preocupación apuntada en un trabajo anterior (Muñoz, 2011), en el que señalábamos que las propuestas de reforma urbana tendían a dejar de lado las cuestiones económicas más estructurales o a focalizar su atención en los mercados de suelo y vivienda ${ }^{12}$, haciendo un recorte analítico de la población urbana en su carácter de "habitantes", es auspiciosa la emergencia de los foros sociales urbanos. Según la Declaración del II Foro Social Urbano (NAPOLI, 2012), ${ }^{13}$ se observa que sí tienen en cuenta la lucha por el trabajo y la vida tanto de los seres humanos como de la naturaleza lo que implica, según sus propios términos, la distribución de las riquezas, la soberanía alimentaria, la defensa de los bienes comunes, la problematización de lo que denominan la globalización financiera y la democracia participativa, entre otras.

Los foros urbanos de distintas ciudades también ilustran esta tendencia: el de Quito, por ejemplo, se plantea los lemas de "otra ciudad es posible" y de "otra economía es posible" de forma inseparable, en un contexto en el que incluso se está problematizando el buen vivir tanto a nivel urbano como rural en conjunto y simultáneamente. ${ }^{14}$

\section{EL SECTOR INFORMAL URBANO: LA HEGEMONÍA QUE PERDURA}

Al contrario de lo que se evidencia en la complejización e interesantes evoluciones reseñadas en el punto anterior sobre las prácticas económicas urbanas tanto reivindicativas como de construcción de satisfactores para la satisfacción de necesidades sociales, a nivel teórico y como fundamente de muchas políticas, seguimos encontrando que perdura la hegemonía del enfoque de la informalidad que no ven, niegan o intenta deslegitimar tales prácticas.

Si bien, según Tokman (1995), existen varios enfoques del sector informal urbano (PREALC, neoPREALC, empiricista, legalista, etc.), estos definen a las unidades económicas casi de la misma forma y, en general, es remarcable la pérdida de especificidad urbana.

El concepto de informalidad y el par formal/informal fue propuesto por Keith Hart a principios de la década de los setenta a partir de un trabajo etnográfico realizado en África. Sin embargo, años después, criticó tal categoría por ser estática y dualista $^{15}$. El concepto fue rápidamente apropiado por la OIT y adaptado al ámbito latinoamericano a través de la PREALC, siendo hasta la actualidad sustento de políticas públicas dirigidas a los sectores populares.

En general, a las unidades económicas denominadas informales -comúnmente categorizadas como "microempresas informales"- se les atribuye una serie de características, como por ejemplo: baja escala; baja capitalización por cantidad de trabajadores (habiendo casi una obsesión por el indicador $\mathrm{K} / \mathrm{L}$ ); bajo nivel tecnológico o muy simplificado; formar parte de mercados competitivos o ser la base de la pirámide de oferta de estructuras oligopólicas, en actividades con bajas barreras a la entrada; propiedad de los medios de producción, generalmente, de los miembros-trabajadores (la categoría utilizada es "mano de obra"); ilegalidad parcial o total; generación de excedentes reducidos, todo lo cual produce como resultado una "reproducción simple", debiendo aclarar aquí que se refieren a la reproducción del capital y no a la de la vida. 
En esta línea, en una de las primeras publicaciones sobre el enfoque en Latinoamérica, Souza y Tokman (1976: 387) plantean la existencia de un mercado de trabajo formal estratificado que permite visualizar el sector informal como el último eslabón en la jerarquización de la actividad económica establecida por la heterogeneidad estructural. Asimismo y, definen el sector informal como

[...] el conjunto de personas ocupadas en el servicio doméstico, los trabajadores ocasionales, los trabajadores por cuenta propia y los patrones, empleados, obreros y trabajadores familiares ocupados en empresas cuyos efectivos no excedan de cuatro personas [...] Se utiliza también como criterio alternativo incluir en este sector a las personas que perciben ingresos inferiores a un cierto mínimo, por lo común el mínimo legal, bajo el supuesto de que las actividades de baja productividad que caracterizan al sector informal generan, asimismo, ingresos reducidos.

A pesar de la diversidad de prácticas bajo la informalidad, este enfoque tiene una clara orientación para las políticas públicas. La mayoría de las políticas que se derivan de sus recomendaciones son de corte microeconómico, con énfasis en la simplificación de la burocracia estatal para lograr la formalización; separación del negocio y la familia; capacitación y crédito destinado a los microempresarios, con miras a lograr "el salto de la microempresa informal a la empresa moderna". ${ }^{16}$ No cualquier capacitación ni crédito, sino modalidades específicas y de un alcance muy acotado, con una fuerte complementariedad con las microfinanzas, especialmente, el enfoque minimalista. ${ }^{17}$

Desde hace años, varios autores vienen criticando estas propuestas. Se destacan, entre estos, los planteos precursores de Santos (1975), Coraggio (1994, 1995) y Pradilla (1995). Además de la cuestión estática y dual ya mencionada por el propio Hart, otra de las cuestiones que se le suele criticar es la inutilidad de la "informalidad" como categoría analítica, ya que bajo dicho paraguas pueden encontrarse actividades y racionalidades tan heterogéneas como las actividades de la economía popular, de la economía criminal, las de empresas capitalistas que evaden impuestos, e incluso hasta los propios estados que precarizan el trabajo respaldándose en las normas de la flexibilización laboral. Es tan cierto esto como que muchas de las iniciativas de la economía popular son totalmente formales. Trabajos más recientes del enfoque informal han reconocido esta situación, en sus propios términos:

[...] ni el sector informal opera absolutamente "en negro", ni su opuesto, el sector moderno, lo hace con un irrestricto apego a la legalidad. Predominan en cambio las llamadas áreas grises $[\ldots]$ : se cumple con ciertos requisitos de registro, pero no se pagan los impuestos; se observa parte de las regulaciones laborales, pero no todas. Tal situación es extensiva a las actividades modernas, particularmente en países con escasa disciplina fiscal y reducida capacidad de fiscalización; obviamente, en ellas la ilegalidad absoluta es casi inexistente, pero la legalidad parcial es también significativa. (TOKMAN, 2001: 16-17).

Sin embargo, en vez de profundizar sobre los grises, siguen trabajando sobre la categoría de informalidad tanto a nivel de las empresas como del trabajo.

Por otro lado, propuestas como los de H. de Soto (2000) exacerban la visión neoliberal del enfoque, al sostener que titularizar la propiedad de ciertos "activos de los pobres" ${ }^{18}$ resuelve problemas de los microempresarios, por ejemplo, el acceso
16 Esta expresión surge del trabajo de campo realizado en mi tesis de maestría: Muñoz, R. (2007) "Alcance de las microfinanzas para el desarrollo local. Microcrédito en el Conurbano Bonaerense: el Banco Social Moreno y Horizonte" en Muñoz, R.; Verbeke G.; Carbonetti, C.; Ozomek, S. (2007). Las finanzas y la economía social. Experiencias argentinas. Buenos Aires, Editorial Altamira.

17 Para más detalles al respecto, se sugiere leer la tesis mencionada en la nota al pie anterior. 
18 Un análisis profundo y detallado de este enfoque se encuentra en la compilación de tesis de la Maestría en economía social plasmada en: Coraggio, J. L.; Costanzo, V. (Eds.). Mentiras y verdades del "capital de los pobres". Perspectivas desde la economía social y solidaria. Colección Lecturas de economía social. Los Polvorines, IMAGO MUNDI-UNGS. al crédito formal por pasar a contar con garantías reales. $\mathrm{Al}$ respecto, enfoques más interesantes sobre la informalidad urbana, que ponen el acento en las cuestiones del hábitat popular, sostienen que existe un incumplimiento de estas promesas. En esa línea, Calderón (2003) asegura que, en Perú, las familias con título de propiedad prácticamente siguen sin acceder al crédito formal.

Otra cuestión crítica es que este enfoque está pensando en la inclusión de los informales en los mercados que ya los excluyeron y donde no caben todos, salvo en condiciones netamente desfavorables. Como plantea Vázquez (2010), se da un debate entre modernizar y tolerar. Desde la vertiente de Tokman (2001), se diagnostica que las microempresas informales deben adecuarse a las empresas modernas, focalizando el problema en los sujetos que deben ser ayudados para realizar esta transformación con las políticas señaladas más arriba. En cambio, para Portes y Haller (2004) el problema está en la estructura económica capitalista periférica y no ven posibilidades de mejora para estos sectores.

Desde la perspectiva de la ES, en definitiva, es preciso poner énfasis en la negación por parte de este enfoque del pluralismo económico y en su permanente presión sobre los sectores populares para que modifiquen sus prácticas según la racionalidad capitalista. En este sentido, una de sus recomendaciones privilegiadas es la separación de los gastos, inversiones y patrimonio entre "la microempresa" o "el negocio" y la familia que lo lleva a cabo, lo que, según nuestro punto de vista, atentaría contra su propia sostenibilidad.

De todas formas, la vigencia de la hegemonía de este enfoque perdura. Quizá una ilustración preocupante de esto sea la aceptación que tuvo por parte de la CEPAL al aggiornar la tesis de heterogeneidad estructural de Prebisch, planteando la existencia de tres velocidades. Los países de la región presentan una estructura productiva más heterogénea que en el pasado y que la de otras economías en desarrollo como las de Asia oriental. Esta situación se puede representar, de manera esquemática mediante un modelo de economía de [...] tres velocidades, según la modalidad de inserción legal y el tamańo de las empresas que la conforman:

- Un primer grupo está constituido por las empresas informales que, por su estructura y capacidad, presentan la productividad relativa más baja y operan en un contexto que les ofrece pocas oportunidades de desarrollo y aprendizaje.

- El segundo está integrado por las empresas formales medianas y pequeñas que, a su vez, tienen dificultades para acceder a los recursos, especialmente financieros, $\mathrm{y}$ a determinados mercados de factores que les permitirían desarrollar su capacidad de competir.

- El último grupo abarca a las grandes empresas, nacionales y extranjeras, que muchas veces tienen un nivel de productividad cercano al de la frontera internacional, pero cuentan con escasos encadenamientos al resto de la economía nacional y, en algunos casos, exhiben baja capacidad de generación de innovaciones. (CEPAL, 2004: 90).

A pesar de las fuertes críticas a este enfoque y las evidencias empíricas que niegan sus predicciones, manifestaciones como estas dan cuenta de que lamentablemente siguen siendo fundamento de políticas públicas y encontrando eco en la academia. 


\section{LA TEORÍA DE LOS DOS CIRCUITOS Y LA ECONOMÍA SOCIAL: UN DIÁLOGO PENDIENTE}

Planteando una profunda crítica al enfoque del sector informal urbano, la teoría de los dos circuitos de la economía urbana en países subdesarrollados fue desarrollada por Milton Santos desde inicios de la década de los setenta (SANTOS, 2008 [1975]) y es permanentemente complejizada y actualizada, fundamentalmente, por geógrafos brasileros, entre los que se destacan los trabajos de Silveira (por ejemplo, 2007, 2004).

Esta teoría considera que existen dos circuitos de la economía urbana, tratándose de un "[...] verdadero paradigma nuevo de la geografía urbana y de la planificación en los países subdesarrollados" (SANTOS, 2008: 38). Se trata de dos circuitos económicos que surgen de la coexistencia de varias divisiones territoriales del trabajo. Por un lado, el circuito superior (CS) que surge como resultado directo de la modernización tecnológica y consiste en las actividades creadas en función de los progresos tecnológicos de las personas que se benefician de ellos. Por otro lado, el circuito inferior (CI) que surge como resultado de la misma modernización pero como resultado indirecto; en él se desenvuelven los individuos que solo se benefician parcialmente o no se benefician de los progresos técnicos recientes y de las actividades ligadas a ellos.

La relación entre ambos es una oposición dialéctica, ambos son opuestos y complementarios, aunque para el circuito inferior, la complementariedad adquiere la forma de dominación o dependencia (SANTOS, 2008; SILVEIRA, 2007). El CI es dependiente del CS y no tiene autonomía de significado (como la propuesta del SIU) porque "[...] no tiene autonomía de existencia en la economía urbana" (SILVEIRA, 2007: 150).

Siguiendo estos trabajos de Silveira, el papel de la tecno-ciencia, de la información y de las finanzas explica la difusión de actividades bancarias, comerciales, industriales y de servicios, frecuentemente orientadas a la exportación, que se localizan en zonas modernas de la ciudad y componen el CS. Como resultado indirecto de esa modernización, surge el CI que se instala allí donde el medio construido urbano se encuentra deteriorado o menos provisto de equipamientos e integrado por formas de fabricación, comercio y servicios sin uso intensivo de capital que satisfacen las demandas de los estratos inferiores. Un bajo grado de capital se inmoviliza para crear, a partir de relaciones técnicas y de organización poco sofisticadas, un número significativo de puestos de trabajo.

A su vez, como fracción indisoluble del CS, se puede identificar el circuito superior marginal (CSM), constituido por formas mixtas pertenecientes tanto a las actividades heredadas como a formas de trabajo emergentes que participan, subordinadamente, de la división del trabajo hegemónica. Amerita aquí tomar un ejemplo que permite ilustrarlos: "Si los objetos vinculados a las telecomunicaciones y a la reparación de máquinas de base industrial amplían el universo del CSM por la demanda de calificación y de instrumentos específicos, la reparación de buena parte de la actual base material doméstica fundada en el consumo globalizado (como los electrodomésticos y a veces los vehículos) se refugia frecuentemente en el circuito inferior" (SILVEIRA, 2004:3).

A pesar de las escuetas presentaciones de las categorías centrales de la T2C y la ES, es notable la potencialidad que puede tener ponerlas en diálogo y llama la 
19 Para una mayor rigurosidad, cada uno de los puntos de encuentro y discusión que señalamos a continuación, requieren una mayor ilustración y fundamentación. Sin embargo, por razones de espacio y por tratarse de una primera exploración, consideramos que alcanza para dar cuenta de la importancia del dialogo que se ha planteado.
20 El número de página corresponde a la introducción del libro, en la versión disponible en: <www.coraggioeconomia.org $>$.

21 En los últimos años, desde el Área de Sistemas económicos urbanos del ICO-UNGS, se está encarando la problematización de la economía de las ciudades desde la perspectiva de la ES y organizando actualmente dos volúmenes sobre la temática, con contribuciones originales de autores destacados del campo en América Latina. atención que aún esto no se haya encarado ${ }^{19}$. Dicha potencialidad proviene del hecho que ambas teorías tienen una fuerte raigambre latinoamericana, con un diagnóstico que se preocupa tanto por "lo económico" como por "lo social" y "lo político", viendo que ambas rompen con la usual y artificial separación entre dichas dimensiones.

A su vez, ambas consideran las causas y no solo las consecuencias de las problemáticas urbanas, entendiendo que es el sistema capitalista el que empuja a las peores condiciones de vida y de trabajo de los sectores populares y, por tanto, ambas se han ocupado de criticar al enfoque del SIU, en particular, su visión dual de los procesos socioeconómicos.

Por otro lado, es relevante observar que ambas son multiescalares, considerando tanto la escala barrial, como la urbana, regional, nacional y global, diferenciándose de la oposición más generalizada local/global. Al respecto, es preciso recordar que cuentan con definiciones propias del espacio, alejándose de las nociones tanto administrativo/ políticas como de la economía espacial que sigue proponiendo un espacio euclidiano bien distinto al del "territorio" realmente existente.

Es destacable, por último, que las dos tienen capacidad de incidencia para ayudar a transformar el campo de la EU al constituirse en verdaderas escuelas, por contar con referentes académicos comprometidos con la permanente actualización y complejización de sus elaboraciones teóricas y con la difusión y formación.

Partiendo de estos puntos de encuentro, que a nuestro juicio no son para nada insuficientes, señalamos provocativamente algunas cuestiones que pueden llevar a interesantes discusiones.

\section{Sobre LA ESPECIFICIDAD URBANA}

Vale la pena señalar, en primer lugar, una cuestión sobre la génesis y evolución de la corriente de ES en el marco de la cual trabajamos. En los propios términos de Coraggio: "[...] a medida que avanzábamos en la elaboración, se fue dando un movimiento conceptual que sigue esta secuencia: crítica del concepto empiricista del sector informal urbano _ economía popular urbana _ variantes de ESS _ economía del trabajo" (CORAGGIO, 2004: 5), ${ }^{20}$ siendo la economía del trabajo una construcción posible de una economía no organizada según la lógica del capital sino de la reproducción de la vida a partir de la desalienación del trabajo y, como decíamos al inicio, la ESS un campo de prácticas orientado por la reproducción ampliada de la vida de todos.

De esta forma, si bien la ES surge ocupándose fundamentalmente de la problematización de la economía y la sociedad urbanas, se fue distanciando de la ciudad y transitando hacia una teoría más general. ${ }^{21}$

En cambio, la T2C surge de las economías urbanas de los países subdesarrollados y en ese ámbito ha trabajado desde su génesis. Creemos que esto trae aparejadas las ventajas de toda especialización y que con los esfuerzos de actualización que se siguen realizando, se lograría captar especificidades y transformaciones de las ciudades que pueden perderse en la ES. 


\section{Sobre las PluRALIDADES DE RACIONALIDADES ECONÓMICAS Y LAS UNIDADES} DE ANÁLISIS PRIVILEGIADAS

La ES reconoce en la economía popular que puede darse una racionalidad económica alternativa a la privada capitalista y la pública en tanto que, entre otras cuestiones, se da una unidad entre la producción y la reproducción estando la primera sujeta a la segunda y concebida como un medio y no un fin en sí misma. De esto se desprende que la unidad de análisis jerarquizada por la ES sean las unidades domésticas populares y los emprendimientos asociativos. No obstante, vale la pena aclarar, no se trata de una idealización de la economía popular como pueden sostener otras corrientes de la ES, sino que se observa la heterogeneidad de lógicas vigentes y no se le adjudica ex ante una estructuración de las relaciones de determinada calidad (reciprocidad, solidaridad, etc.).

Si bien la T2C subraya la dependencia del trabajo de los agentes del CI y el CMS para su vida y analiza cómo se prioriza el trabajo por sobre el capital, planteando irónicamente un comportamiento "irracional" (desde el punto de vista hegemónico claro está), ${ }^{22}$ no ve una racionalidad económica necesariamente alternativa (al menos, de lo que pudimos captar en estas primeras lecturas que estamos realizando). A su vez, la teoría no trata la salida asociativa y las unidades domésticas populares como un sujeto de análisis, al priorizar a las empresas de pequeña escala y, de hecho, subrayando un distanciamiento con "[...] la literatura específica [en la que] es frecuente [encontrar] la asociación entre esta economía de escaso capital, tecnología y organización, y el empleo familiar" (SILVEIRA, 2007: 156). Esta distancia se sostiene en datos que revelan que en el CI y en el CMS no predominan las relaciones de parentesco, salvo en algunas ramas de la producción determinadas entre las que se destaca la textil. ${ }^{23}$

Por su parte, una encuesta realizada desde la perspectiva de la ES en ciudades de Argentina en 2005 muestra lo contrario (CORAGGIO; FEDERICO SABATÉ, 2010). ${ }^{24}$ Lamentablemente, en la publicación no se encuentra desagregado el nivel de relación de parentesco por rama de actividad, lo que sería interesante de indagar.

Más allá de estas diferencias en los resultados, ambos evidencian la importancia de realizar análisis territoriales y sectoriales que aborden todos los agentes socioeconómicos realmente existentes, sin forzar la realidad como suelen hacer las investigaciones del SIU, de forma tal de poder alcanzar diagnósticos más acabados sobre la economía urbana realmente existente.

\section{Sobre el tratamiento de las RElaciones ENTRE loS Distintos CiRCUitos/ SUBSISTEMAS Y LAS CARACTERÍSTICAS DE LOS TRABAJOS EMPÍRICOS LLEVADOS ADELANTE POR CADA TEORÍA}

Ambas teorías plantean las complejas relaciones entre los circuitos (según la categoría de la T2C) o subsistemas (ES), las asimetrías de poder que tienen el CI y el CMS o la economía popular con respecto a las empresas capitalistas y al Estado. Sin embargo, analizando algunos de los últimos trabajos empíricos llevados adelante por ambos, se observa que la ES pone mayor acento en la economía popular o en las experiencias de ESS que en el trabajo asalariado o el papel de las corporaciones globales. Por su parte, la T2C realiza trabajos empíricos de mayor alcance, que logran
22 Como explica Silveira: "Destinar una proporción mayor de los rendimientos al pago de mano de obra en lugar de aumentar y modernizar el capital fijo (sobre todo localización y maquinarias) no deja de ser, en el período actual, una producción de irracionalidad. Una buena localización, capaz de atraer grupos de población de mayor poder adquisitivo, así como la compra de equipos más modernos, que ahorran mano de obra, sus cargas sociales y los eventuales conflictos, serían, ciertamente, formas de producción de racionalidad. Esencia de las políticas del poder público y de las grandes empresas, esa racionalidad se revela limitada, sobre todo en las grandes metrópolis [...] Sin embargo ¿cuál es la racionalidad de una economía urbana que desprecia, como en Salvador, cerca del $30 \%$ de su PEA? El hecho es que cada día hay un número menor de actividades y de empleos relacionados con esa racionalidad hegemónica y esto es más visible en las grandes ciudades de Brasil. Frente a esa realidad, una enorme parte de la población debe encontrar una actividad" y un lugar dentro de la ciudad "que sea capaz de permitir su supervivencia. Es una economía de abajo hacia arriba (SANTOS, 1996; 2000) que parte del principio de que los más pobres, los vecinos, los demás trabajadores podrán consumir los productos y servicios ofrecidos. Es una cooperación que se completa en la contigüidad $y$, por lo tanto, no necesita ser fluida, ni veloz, ni competitiva. Esa cooperación puede así ser lenta, y por eso es vista como irracional" (SILVEIRA, 2004: 6-7).

23 Los resultados que presenta Silveira son los que siguen: "Del total de las encuestas realizadas, $63,4 \%$ señalaron que no utilizan mano de obra fa- 
miliar, aunque la distribución del fenómeno es bastante heterogénea. Por ejemplo, en las fábricas de tejidos y confecciones de Fortaleza, el empleo familiar es frecuente para más de la mitad de las firmas investigadas. Semejante es la situación de los negocios de materiales de construcción y de los comercios de herramientas $y$ herrajes en el centro antiguo de Campinas. Cerca de la mitad de los agentes del $\mathrm{Cl}$ como mercados y almacenes, casas de variedades y mercerías, bares y restaurantes, servicios de reparación, alquiler de videos, cerrajeros, ópticas y relojerías contratan mano de obra familiar. Ese tipo de empleo es menos relevante en las ciudades de San Pablo, Rio de Janeiro, Porto Alegre y Goiânia. En San Pablo menos del 30\% de las firmas encuestadas utiliza mano de obra familiar (SILVEIRA, 2007: 156).

24 Las relaciones de parentesco en 611 emprendimientos asociativos mercantiles son del $59,1 \%$, en un universo compuesto por varias ramas de la producción entre las que se destacan la producción textil $(23,4 \%$ de los emprendimientos), seguida de cerca por la elaboración de alimentos (22,6\%) (CORAGGIO; FEDERICO SABATÉ, 2010: 47; 37). La relativa predominancia textil no parece de todas formas estar sesgando los resultados de la muestra no representativa, en virtud de que ni llega a constituirse en un cuarto de la misma. cubrir varias ramas sectoriales y varias metrópolis brasileñas, teniendo en cuenta desde el fenómeno de las moto-taxis hasta la topología de las corporaciones globales.

Sin embargo, vale tener en cuenta las diferentes condiciones de producción académica que existen en los países que estamos tomando como referencia (Argentina y Brasil), siendo el bajo alcance empírico una característica de toda la producción académica sobre la economía de la RMBA como seńalan Palma y Borello (2010).

\section{Sobre el papel de las políticas públicas}

Del análisis realizado pareciera que la T2C pone mayor énfasis en la comprensión de los procesos socioeconómicos desde las actividades empresariales a lo largo de los distintos circuitos que de las políticas públicas. A su vez, se observa una lectura de las intervenciones del Estado en favor del circuito superior. En el caso de la ES, vemos interés tanto en los agentes populares como en el Estado, con una visión del mismo como regulador de la relación capital/trabajo, lo que implica también la búsqueda del mantenimiento de las condiciones de gobernabilidad deseables en intervenciones siempre contradictorias, con márgenes de acción y espacios en los que se da lugar a prácticas más participativas (como puede ser el presupuesto participativo) y hasta la co-construcción de las políticas. En este marco, es precisamente en esas interfaces en las que el Estado, junto con una sociedad organizada, lleva a cabo acciones que favorecen a la economía popular urbana y las experiencias de ESS, con una permanente crítica a las políticas sociales focalizadas y a la necesidad de formulación e implementación de políticas socioeconómicas (por ejemplo, CORAGGIO, 1999; HINTZE, 2007).

Podríamos seguir desarrollando más puntos de encuentro y desencuentro, buscando las posibilidades de construcción teórica articulada entre ambos abordajes. Sin embargo, creemos haber ilustrado lo suficiente como para dar sustento al puntapié inicial para encarar dicho diálogo.

\section{REFLEXIONES FINALES}

La institucionalización de la ESS en América Latina es evidente, así lo indican diversas prácticas de ESS, políticas y hasta nuevas constituciones como en Ecuador y Bolivia. Por su parte, a nivel de las prácticas reivindicativas sobre lo urbano, se da una creciente complejización de la mirada sobre la economía de las ciudades, antes muy concentrada en el mercado de suelo y la vivienda.

Desarrollos de esta complejidad siguen marcando la necesidad teórica de avanzar en un tratamiento integrado de forma compleja de los mundos del hábitat y demás esferas reproductivas (como el cuidado, la protección social, el consumo, etc.) y el mundo del trabajo y la producción que se dan en nuestras ciudades.

Sin embargo, se mostró que perdura la hegemonía de la informalidad, a pesar de las críticas, de la falta de evidencia empírica sobre sus predicciones y de la encarnación del proyecto neoliberal en sus interpretaciones teóricas y recomendaciones de política.

Por otra parte, prácticamente no se detectan intercambios entre sus enfoques críticos. Al respecto, este artículo propone exploratoria y provocativamente un diálogo entre la T2C y la ES, llamando la atención sobre el hecho de que a pesar de los varios puntos de encuentro entre ambas, existe una cuasi ignorancia recíproca. En 
posible, una vez más, que los recortes disciplinarios sean la principal causante. Si bien la ES se basa en un tratamiento pluridisciplinario de lo económico, en las primeras formulaciones prevalecía la economía, mientras que en el caso de la T2C se ve el predominio de la geografía. Esto plantea la iniciativa de encarar el trabajo académico de forma tal de superar los recortes disciplinarios que operan sobre la ciudad en pos de una necesaria integración analítica.

En esta línea creemos, en particular, que el establecimiento de tal diálogo constituye una deuda del campo y un desafío a encarar para la construcción de mejores diagnósticos sobre nuestras ciudades y, en el mejor de los casos, para concretizar aportes tanto en la formulación y evaluación de políticas como en la creciente complejización de las estrategias de las organizaciones y movimientos involucrados en las disputas por el derecho a la ciudad y las mejores condiciones de trabajo y de vida de los seres humanos y la naturaleza.

\section{REFERÊNCIAS BIBLIOGRÁFICAS}

CALDERÓN, J. Formalización de la propiedad, cultura registral y crédito. In: ABRAMO, P. (org.) A cidade da informalidade. $O$ desafio das cidades latino-americanas. Rio de Janeiro, Lincoln Institute, Sette Letras/Faperj, 2003.

CEPAL (2004). Desarrollo productivo en economías abiertas. Secretaría ejecutiva.

CORAGGIO, J. L. Territorio y economías alternativas. In: ACOSTA, A.; MARTINEZ, E. (eds.) Economía social y solidaria. El trabajo antes que el capital. Quito, Ed. Abya Ayala, 2011a.

CORAGGIO, J. L. La presencia de la ESS y su institucionalización en América Latina.

Contribution a les Etats généraux de l'ESS” 17-19 juin, Paris; disponible en: <www.

coraggioeconomia.org $>$.2011b

CORAGGIO, J. L. Introducción. In: De la emergencia a la estrategia. Más allá del alivio de la pobreza. Buenos Aires, Espacio Editorial. Disponible en: <www.coraggioeconomia.org>., 2004. CORAGGIO, J. L. Política social y economía del trabajo. Alternativas a la política neoliberal para la ciudad. Buenos Aires, UNGS-Miño y Dávila, 1999.

CORAGGIO, J. L. Economia Urbana: la perspectiva popular. Quito, Instituto Fronesis, 1994. CORAGGIO, J. L.; SABATÉ, A. M. F. (dir.). Emprendimientos socioeconómicos asociativos: su vulnerabilidad y sostenibilidad. Buenos Aires, UNGS, Los Polvorines, 2010.

CORAGGIO, J. L.; PRADILLA, E.; RUIZ, L.; MUNDA, M. (1995). Más allá de la informalidad. Quito, Centro de Investigaciones CIUDAD.

CUERVO, L.M. (2005). El falso espejo de la ciudad latinoamericana. Serie gestión pública $\mathrm{N}^{\circ}$ 52. ILPES - CEPAL.

DE SOTO, H. (2000). El misterio del capital. Lima, El comercio.

FERNANDEZ, V. R.; VIGIL, J. I. (dir.) Clusters y desarrollo regional en América Latina. Reconsideraciones teóricas y metodológicas a partir de la experiencia argentina. Buenos Aires, Miño y Davila/Universidad Nacional del Litoral, 2009.

HINTZE, S. La política es un arma cargada de futuro. La economía social y solidaria en Brasily Venezuela. Buenos Aires, eds. CLACOS-CICCUS, 2010.

HINTZE, S. Politicas sociales argentinas en el cambio de siglo. Conjeturas sobre lo possible. Buenos Aires, Ed. Espacio, 2007.

MUÑOZ, R. Alcances y desafíos de la economía popular urbana. Reflexiones a partir de análisis sectoriales y casos de estudio de la RMBA. In: URQUIETA, P. (coord.) Ciudades en transformación. Disputas por el espacio, apropiación de la ciudad y prácticas de ciudadanía. CIDES-UM-
Ruth Muñoz é Investigadora docente del Área Sistemas Económicos Urbanos del Instituto del Conurbano de la Universidad Nacional de General Sarmiento (UNGS). Coordinadora académica de la Maestría en Economía Social de la misma universidad. E-mail: <rmunoz@ungs.edu.ar>. 
SA. La Paz, Plural Editores, 2011.

MUÑOZ, R. Políticas económicas poscrisis. Aprendizajes y desafíos a partir de la institucionalización de la economía social y solidaria en América Latina. En el libro del Colloque International Penser et construire de nouveaux référentiels écononomiques, Ministerio Delegado de la Economía Social y Solidaria. Bercy, París, 5 de febrero de 2013.

PALMA, C.; BORELLO, J. Relevamiento de Investigaciones y estado del arte de los temas abordados por el Área Sistemas Económicos Urbanos. Buenos Aires, ICO-UNGS, 2010.

PORTES, A.; HALLER W. La economía informal. CEPAL. Santiago, Serie Politicas Sociales, n. 100, 2004.

SANTOS, M. [1975]. O espaço dividido. Os dois circuitos da economia urbana dos países subdesenvolvidos. São Paulo, Editora da USP, 2008.

SILVEIRA, M. L. Metrópolis brasileńas: un análisis de los circuitos de la economía urbana. EURE (Santiago), n. 100, 2007.

SILVEIRA, M. L. Globalización y circuitos de la economía urbana en ciudades brasileñas. Cuadernos del CENDES. v. 21, n. 57, 2004.

SOUZA, P. R; TOKMAN, V. El sector informal urbano en América Latina. Revista Internacional del Trabajo, v. 94, n. 3, 1976.

TOKMAN, V. Introducción. In: TOKMAN, V. (comp.) El sector informal en América Latina. Dos décadas de análisis. Consejo Nacional para la Cultura y las Artes, México, 1995.

TOKMAN, V. De la informalidad a la legalidad. Introducción. In: TOKMAN, V. (coord.) De la informalidad a la modernidad. Santiago de Chile, OIT, 2001.

VÁZQUEZ, G. Hacia una sostenibilidad plural de los emprendimientos asociativos de trabajadores autogestionados. Perspectivas y aportes conceptuales desde América Latina. Tesis de Maestría en Economía Social (ICO-UNGS), 2010. Disponible en: <www.ungs.edu.ar/areas/pos_tesis/104/>.

VUOTTO, M. El cooperativismo de trabajo en Argentina. Contribuciones para el diálogo social. Lima, OIT, 2011.

A B S T R A C T The urban economy has been hardly influenced by spatial proposals and economicism. In Latin America, while the institutionalization of social and solidarity economy of the last few years shows a growing economic pluralism, in the urban economy field persists the hegemony of urban informal sector perspective, and its pressures of transforming in capitalist every popular economy practice. Seeking for recognition of social economy in this field, the article succinctly illustrates social and solidarity institutionalization in the region and suggests a provocative exploration to put in dialogue social economy with two circuits of urban economy theory. Such a dialogue is considered as a debt, which we understand could contribute to dispute the field, to get better explanations and also to transform in a progressive sense the economy of our territories.

K E Y W O R D S : urban: economy; two circuits of urban economy; social economy; social and solidarity economy; popular economy 